\section{Advanced glycation end products}

K. J. Lackner ${ }^{1}$ und D. Peetz ${ }^{2}$

${ }^{1}$ Institut für Klinische Chemie und Laboratoriumsmedizin, Universitätsmedizin Mainz, Mainz, Deutschland

${ }^{2}$ Institut für Labormedizin, Helios Klinikum Berlin-Buch, Berlin, Deutschland

Synonym(e) AGE-Proteine; AGE

Englischer Begriff Advanced glycosylation (glycation) end products

Definition Endprodukte der nicht enzymatischen Reaktion von reduzierenden Zuckern mit Aminogruppen von Peptiden, Lipiden oder Nukleinsäuren.

Bei der Bildung von ,advanced glycosylation endproducts" (AGEs) durch Maillard-Reaktionen erfahren die als Ketoamine gebundenen Zuckerreste auf Proteinen komplizierte Umlagerungen, die zu den dargestellten Endprodukten führen und teilweise mit Quervernetzungen einhergehen, z. B. durch Pentosidin (rot) (aus: Löffler und Petrides 1997):

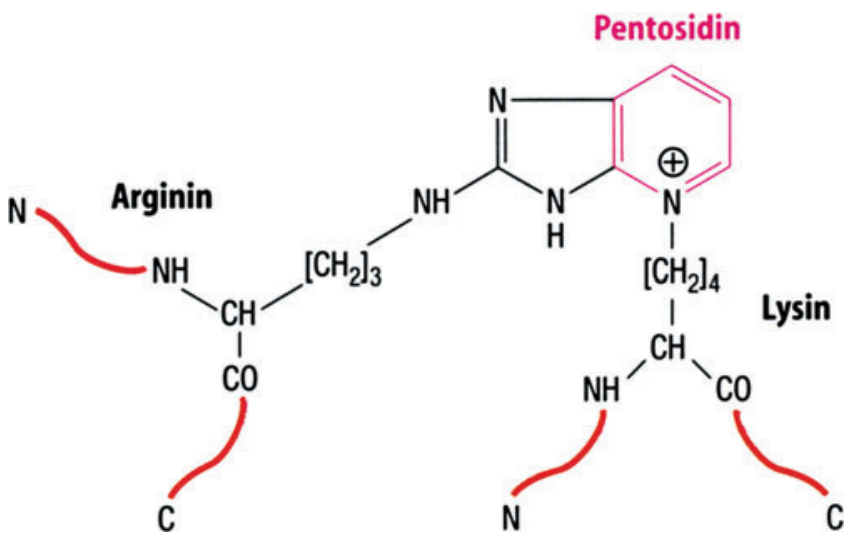

Beschreibung Bereits im Jahr 1912 von L.C. Maillard (1878-1936) beobachtete Reaktion von Aminosäuren mit Zuckern. Über eine Serie von Zwischenprodukten (Schiff'sche Basen, Amadori-Produkte) können Zucker mit Aminogruppen reagieren. Im Ergebnis kommt es zur Bildung von stabilen, kovalenten Addukten, die sich in ihrer Funktion und Struktur z. T. signifikant von ihren nativen Vorläufern unterscheiden. Da der Prozess Tage bis Wochen in Anspruch nimmt, sind vor allem langlebige Moleküle (z. B. Kollagen oder andere Matrixproteine) betroffen. Eine Reihe von immer wieder auftretenden und strukturell aufgeklärten Verbindungen entsteht dabei. Bei Proteinen gehören dazu Pentosidin, $N^{\varepsilon}$-Carboxymethyl-Lysin, die unter oxidativen Bedingungen aus glykierten Proteinen entstehen. Dabei treten intra- und intermolekulare Crosslinks auf. Weitere reaktive Verbindungen bei der Entstehung von AGEs sind Glyoxal, Methylglyoxal und 3-Deoxyglukose, die Dimere mit modifizierten Lysinen bilden können. Man hat spezifische Rezeptoren für AGEs bzw. glykierte Proteine gefunden (RAGE), die relevante Einflüsse auf zelluläre Funktionen haben. Den AGEs wird große Bedeutung in der Pathogenese zahlreicher chronischer Erkrankungen wie der Atherosklerose, neurodegenerativer Erkrankungen, den Komplikationen der Niereninsuffizienz etc. beigemessen. Diagnostisch spielen vor allem glykiertes Hämoglobin und Albumin eine Rolle.

\section{Literatur}

Brownlee M (1995) Advanced protein glycosylation in diabetes and aging. Annu Rev Med 46:223-234

Löffler G, Petrides PE (1997) Biochemie und Pathobiochemie, 5. Aufl. Springer, Heidelberg/Berlin/New York

Singh R, Barden A, Mori T et al (2001) Advanced glycation endproducts: a review. Diabetologia 44:129-146 